kleinerer Dosis, $0,003 \mathrm{~g}$, traten boi dieser vorläufigen Untersuchung folgende,-vielleicht interessante, Erscheinungen auf:

"Der Blutstrom verlangsamt sich und die Zahl der Herzschläge vermindert sich auf die Halfte, um später die Anfangsstärke von nouem zu orreichen, wenn das Chlorhydrat der krystallisierten Base injiziert wurde, und es tritt keine äufserlich bemerkbare Modifikation ein, wenn dieselbe Menge des Chlorhydrats des flüssigen Alkaloïds injizirt wurde. Die Beweglichkeit ist fast gänzlich aufgehoben bei dem Frosch, dem das Chlorhydrat des krystallisierten Alkaloids injiziert wurde.

Sie ist nur modifiziert bei dem Frosch, dem das andere Chlorhydrat injiziert wurde und trotzdem ist die Störung, im Gegensatz zu dem andern, nur von kurzer Dauer, da der Frosch von Neuem Spränge wie im natürlichen Zustande, ausfuhren kann."

Auch diese Untersuchungen werden fortgesetzt, da sie vielleicht Beziehungen zwischen der physiologischen Wirkung und der Konstitution der Alkaloide ergeben.

Ich beschränke mich auf diese Schlisss, ohne viele andere, bereits ausgeführte Untersuchungen zu veröffentlichen, die noch vervollständigt werden sollen, weil bei diesem Gegenstand der Alkaloïde die Litteratur lehrt, dafs man sehr vorsichtig sein mufs, wenn man nicht den hereits verwickelten Gegenstand noch mehr komplizieren will.

Ich begnüge mich also, mit dieser Arbeit den Gegenstand aufgeklärt und grundlegende Thatsachen für die fernere Untersuchung festgestellt zu haben.

\title{
Ueber das ätherische Oel der Wurzel von Aspidium filix mas.
}

\author{
Von Dr. Alex Ehrenberg. $\left.{ }^{1}\right)$
}

(Eingegangen. den 10. März 1893.)

Bei der ungemein häufigen Anwendung, welcher die Wurzel des Wurmfarns und besonders das aus dieser dargestellte ätherische Extrakt sich als Bandwurmmittel zu erfreuen haben, wird es als ein

1) Ol. aethereum. Filicis mar., welckes $z u$ weiteren klinischen Versuchen einladen dürfte, ist zu beziehen von E. Merck, chem. Fabrik, Darmstadt. 
grofser Mifsstand empfunden, dafs die Wirkung dieser Präparate keine gleichmälsige ist. Es ist vorgekommen, dafs bei Verabreichung des Extraktes in Dosen, die bei dem einen Individuum kaum eine Wirkung zu äufsern im Stande waren, bei anderen Personen heftige Vergiftungserscheinungen - sogar mit letalem Ausgange - beobachtet werden konnten. Es lassen sich derartige Beobachtungen ja zum Teil auf die grölsere oder geringere Empfindlichkeit einzelner Individuen gegen gewisse Stoffe setzen; zum weitaus grölsten Teile wird aber doch der geringere oder grölsere Gehalt des Präparates an wirksamen Bestandteilen den Grund für diese Erscheinungen bilden; es steht damit in voller Uebereinstimmung, dals gerade die Filixwurzel je nach der Jahreszeit, in welcher sie gesammelt wird, eine verschiedene Wirksamkeit entfaltet, worauf auch in der Forderung der Pharmacopoea Germ. III. Rücksicht genommen ist, dahin lautend, dal's nur im Herbst gesammelte Wurzel zur Verwendung zu ziehen sei.

Aber eine derartige Forderung bietet durchaus keine Gewähr für den gleichen Ausfall der Droge, denn es werden in gleicher Weise, wie die Jahreszeit, auch Standort und vor allem die Witterungsbedingungen von grofsem Einflufs sein; so finden wir z. B. von $B$ erenger - Feran $t^{1}$ ) die Beobachtung mitgeteilt, dals Wurzeln aus der Normandie fast gar keine Wirkung äufserten, während solche aus den Vogesen und vom Jura sich äufserst kräftig wirkend erwiesen. - Es muls deshalb angestrebt werden, den wirksamen Bestandteil selbst $\mathrm{zu}$ isolieren und durch genane Dosierung eine immer gleiche Wirkung $\mathrm{zu}$ sichern. Als den wirksamen Bestandteil der Filixwurzel sieht man in neuester Zeit die sogenannte Filixsäure an; bereits $\mathrm{P}$ es chi er im Jahre 1825 und Trommsd o r f f und $O s$ a $n$ bemerkten, dafs sich beim Autbewahren von Filixextrakt ein gelblicher, körniger Bodensatz bilde, welcher auch von letzteren gereinigt und untersucht wurde; $\mathrm{Luck}$, im Jahre 1845 ermittelte die Zusammensetzung der Substanz als der Formel $\mathrm{C}_{\mathbf{1 3}}$ $\mathrm{H}_{16} \mathrm{O}_{5}$ entsprechend und als durch die Arbeiten von Liebig, Carlblom und Rulle die Filixsäure als das wirkende Prinzip des Extraktes hingestellt worden war, beschäftigten sich weiterhin

1) Arch. 1886. pag. 1034. - 
mehrere Chemiker in eingehender Weise mit der Untersuchung der chemischen Eigenschaften und Struktur dieses Körpers.

Nach einer Mitteilung von Li ebig ${ }^{1}$ ) soll der Filixsäure die gleiche Wirkung, wie sie dem Extrakte eigen ist, nicht abgehen; Carlblom² sieht in dieser Süure sogar überhaupt den allein wirksamen Stoff der Filixwurzel; $R$ ull $\theta^{3}$ hinwiederum hält die unreine, durch Aufnehmen mit Ammon aus dem Filixextrakt und Ausfällen mit Salzsäure gewonnene Säure für bedeutend wirksamer, als die reine Filixsăure. E. Pou I s s o $n^{4}$ ) kommt auf Grund einer eingehenden Untersuchung zu dem Schlufs, dafs in der That die Filixsäure das anthelmintisch wirkende Prinzip der Filixwurzel sei, dals es aber zwei Modifikationen dieser Säure gebe, welche zu einander im Verhältnifs von Säure zu Anhydrid stehen, leicht in einander überzuführen seien und von denen das Anhydrid (krystallisierte Filixsäure = Filicin) unwirksam sei, während die amorphe Säure - aus orsterem durch Lösen in Alkali und Fällen nit Säure wieder erhältlich -. sehr intensive Giftwirkungen zeige.

Gra e $f^{5}{ }^{5}$ ) dagegen konnte mit Kalt filicinicum Cysticercen im Auge nicht entfernen und Prof. $K$ o b e $\mathrm{t}$, welchem ich sowohl reine krystallisierte Filixsäure, als auch reine a $\mathrm{m}$ orphe Săure zu Versuchen übermittelte, teilte mir früher mit, dafs die krystallisierte Säure absolut keine Wirkungen gezeigt hätte, was ja mit den Angaben $P$ o ulsson's übereinstimmen würde, teilt mir aber auch hinsichtlich der Wirkung der reinen anırphen Säure mit:

„Die amorphe Filixsaure wurde einem mit Band wurm (Bothriocephalus) behafteten Studierenden nach gehöriger Vorkur in einer Dose von $0,6 \mathrm{~g}$ - nebst Abführmittel 20 Min. später - ganz ohne Erfolg gegeben. Erst sls nach $21 / 2$ Stunden noch $0,6 \mathrm{~g}$ der Säure gereicht wurden, gingen zwei kurze Stückchen Bandwurm, aber nicht etwa der Kopf ab. Dieser Versuch zeigt, dafs die reine Säure - ohne ätherisches Öl - entschieden schwach wirkt. $(1,2 \mathrm{~g}$ Säure mufs als eine ungeheure Dose bezeichnet werden )“

l) Investigationes quaedam pharmacolog. de extract. filicis aether. Dorpat 1857 .

2) Ueber die wirksamen Bestandteile des ätherischen Farn-Extrakres. Dorpat 1866.

3) Ein Beitrag zur Kenntnis einiger Bandwurmmittel und deren Anwendung. Dorpat 1867.

4) Arch. f. experim. Pathologie und Pharmakologie 1891.

5) Therap. Monatshefte. 
Ich bemerke hierzu noch, dafs diese Säure, sowohl die amorphe, als die krystallisierte Säure, im Laufe der Jahre bei Gelegenbeit dor Herstellung grofser Mengen Filixextrakt in Quanten von ca. $1 \mathrm{~kg}$ hergestellt worden war und dals ein äufserst sorgfältig gereinigtes Präparat, welches alle die von den Anderen der reinen Filixsäure beigelegten Eigenschaften besals, für diese Versuche zur Verfügung gestellt war.

Es darf nach dem im vorstehenden mitgeteilten doch zweifelhaft erscheinen, ob in der That die Filixsäure der Träger der spezifischen Wirkung des Wurmfarns ist, jedenfalls wird man derselben nicht allein die Wirkung zuteilen duirfen. Als wesentliche Stütze für diese Ansicht führe ich noch an, dafs ich seit Jahren auf Wunsch der Abnehmer auch ein sogenanntes geklärtes Filixextrakt dargestellt habe, aus welchem sich bei längerem Stehen keine Filixsäure mehr ausscheidet, und welche bedeutend weniger dieser Säure noch gelöst enthält, als das nach der Vorschrift der Pharmakopoe dargestellte Extrakt; trotzdem besafs dieses Extrakt mindestens die gleiche Wirksamkeit, wie das andere. - Ein aus guter Herbstwurzel dargestelltes Extrakt zeichnet sich vor einem solchen aus Frübjahrswurzel gewonnenen durch einen bedeutend intensiveren, charakteristischen Geruch aus, welchen ich einem grölseren Gehalte an ätherischem Öle zuschriob und suchte ich deshalb za entscheiden, ob nicht dem ätherischen Öle vielleicht dio charakteristische Filixwirkung - wenn nicht allein, so doch zum grölseren Teile - zuzuschreiben sei. Behandelt man das Filixextrakt mit Wasserdampf, so wird das in geringen Mengen vorbandene ätherische öl fortgeführt; derartiges Extrakt sowohl, als auch ein aus vorher von ätherischem Öle befreiter Wurzel hergestelltes Präparat wurde von Herrn Prof. Kobert in Dorpat auf meine Veranlassung untersucht und als bedeutend schwächer wirkend befunden, als normales Filixextrakt; dagegen liels sich zwischen einem normalen Extrakt und einem solchen, welches durch Petroläther von der Filixsäure fast, vollständig befreit war, kein Unterschied in der Wirkung konstatieren. Ueber das ätherische Filixöl habe ich in der Literatur bisher keine Angaben finden können, in der Real- Encyklopädie für die gesammte Pharmacie Bd IV. pag. 355 ist angegeben, dafs die Wurzel ungefähr 0,04 Proz. ätherischen Öls enthalte, bei allen son- 
stigen Mitteilungen, welche sich mit einem Oleum aethereum Filicis beschäftigen, versteht man darunter das ätherische Extrakt. - Ich habe nun grörsere Mengen ätherischen Öles durch Destillieren mit Wasserdampf aus frisch gesammelter, lufttrockener Wurzel dargestellt und dabei gefunden, dals die Ausbeute an Öl auch wesentlich von der Jahreszeit abhängt, in welcher die Wurzel gesammelt wurde. Das Destillat ist stark sauer und enthält neben aufschwimmendem Öle auch noch nicht unbeträchtliche Mengen von Substanz in Lösung; das Destillat wurde mit Äther ausgeschättelt, von welchem die gelösten Anteile sehr leicht der Flüssigkeit entzogen werden, beim Abdestillieren des Äthers bleibt dann das als rohes Filixöl bezeichnete Gemisch zurück; auch durch Cohobiren des Destillates und Aussalzen kann man das Öl fast vollständig gewinnen. Es wurden auf diese Weise erhalten

Aus 100 Ko. April-Wurzel 8 grm. Oel $=0,003$ Proz.

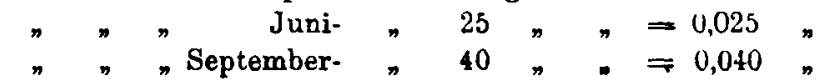

Die Ausbenten an atherischem Öle ans Wurzeln, die im September, Oktober und November gegraben waren, zeigten keine Abweichungen von einander und gaben Werte, die zwischen 0,04 und 0,045 Proz. schwankten. Das ätherische Filixöl ist eine hellgelbe Flüssigkeit von eminent intensivem Filixgeruch, es zeigt bei $15^{0}$ das spezifische Gewicht 0,85 bis 0,86 und geht in der Hauptmenge bei der Destillation zwischen $140^{\circ}$ und $250^{\circ}$ uber, die zwischen 250 bis $350^{\circ}$ übergehenden Anteile sind dunkler gefärbt und zum grofsen Teile Zersetzungsprodukte; es ist indessen auch bei der Destillation im Vacuum kein besseres Resultat zu verzeichnen.

Von dem rohen Filixöl ubermittelte ich Anfangs des Jahres 1892 Herrn Prof. Kobert in Dorpat einige Proben mit der Bitte, zu untersuchen, in wie weit dieses Öl an der wurmwidrigen Wirkung des Filixextraktes beteiligt sei; Herr Prof. Kobert benachrichtigte mich durch private Mitteilung, dafs dem Öle in der That eine wesentliche Wirkung innewohne, worauf ich gröfsere Mengen desselben herstellte und ihm za Versuchen zur Veriügung stellte; als Resultate der eingehenderen Untersuchung teilte mir Herr Prof. K o bert folgendes $\left.\operatorname{mit}:^{1}\right)$

1) Sămtliche hier erwăhnten Präparate wurden durch mich von Seiten der Firma H. Tromm $\mathrm{m}$ d or f $\mathrm{f}$ in Erfurt Herrn Prof. Kobert 
„Das aeth. Filixöl wurde auf seine Wirksamkeit in mindestens 50 Tersuchen geprüft, die sich auf verschiedene Tierarten sowie auf den Menschen beziehen. Da es sich in Wasser nicht oder fast nicht löst, so wurde es den Nährlösungen, in welchen sich Wassertiere befanden, z. T. nach Verdünnung mit Alkohol, z. T. unverdüunt zugesetzt und durch häufiges Schütteln mechanisch verteilt. In alkoholischer Lösung wurde es nur zu solchen Versuchen verwendet, bei welchen die entsprechende (stets geringe) Menge Alkohol an sich sich als nicht schädlich erwiesen hatte. Die Versuche im Eiuzelnen ergaben Folgendes:

Regenwturmer werden durch das Öl schon in grofser Verdünnung

$$
1: 3000 \quad 1: 2000-1: 10 \% 0
$$

gelähmt, ja abgetötet. Das Gleiche gilt in noch höherem Grade von Kaulquappen. Auch bei diesen tritt - selbst wenn das Öl nicht mit Alkohol verdünnt dem Wasser zugesetzt wird - vorher eine Art Narkose ein. Von Taenien wurden solche von Fischen, Katzen und Hunden in passend gewählten und passend temperierten Nährlösungen untersucht. Sie starben bei einer Konzentration des Öles in der Lösung

$$
\begin{aligned}
& \text { ron } 1: 1250 \text { binnen } \quad 21 / 2 \text { Stunden. }
\end{aligned}
$$

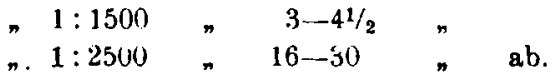

Spulwürmer sind gegen unsere stärksten Gifte nach $W$. vo $\mathrm{S}$ ch roed e r selbst bei tagelanger Einwirkung des Giftes (in der Nahrlösung) fast unempfindlich. Nichtsdestoweniger wurden sie durch der Nährlösung zugesetztes Filixöl bei einer Konz. von $1: 1500$ binnen 3 bis 6 Stunden und bei einer Konz. von $1: 2500$ binnen 18-37 Stunden abgetötet. Wurden sio vorher aus der Lösung genommen, so waren sie starr, erholten sich aber in unvergifteter Nährlösung wieder. Auch bei den Bandwürmern geht dem Absterben ein Stadium der Lähmung vorher. Dieses Stadium tritt bei der Bandwurmkur mit dem Filixextrakt im Darmkanal des Menschen ein, denn der Wurm kommt meist nur betäubt, nicht tot zu Tage. Die aus dem Hecht geschnittenen Jugendzustände von Bothriocepbalus latus, welche sich in Nährlösungen lange halten und sehr echön bewegen, wurden durch das Ol ganz wie Taenien beeinflufst. Nach allen diesen Versuchen mufs das Filixŏl als ein spezifisches Gift für niedere Tiere bezeichnet werden, welches ohne Frage bei der Bandwurmkur mittelst Farnkraut mitwirkt.

Auf gesunde Menschen wirkten innerlich in Gelatinekaspeln genommene einmalige Dosen von 0,75-1,6 Gramm des Öles nicht schädlich oin. Der Kot roch intensiv nach dem Öl.

zur Verfügung gestellt und hatte genannter Hers die Liebenswürdigkeit, die Kesultate seiner Untersuchungen der Firna behufs Verwertung in den wissenschaftlichen Mitteilungen zur Verfägung zu stellen, wo. für ihm an dieser Stelle gedankt sei: ausführlichere Mitteilungen uber diesen Gegenstand wird Herr Prot. $K$ ob ert an anderer Stelle bringen. 
Zieht man in Erwägung, dals ein Extrakt, welches von Filixsïure befreit war, in seiner Wirkung nicht nachweisbar abweicht von einem solchen mit vollem Gebalte an dieser Säure, dafs dagegen nach Entfernung des ätherischen Oeles aus dom Extrakte eine wesentliche Verminderung der Wirksamkeit eintrat und dafs bei Prüfung des Oeles selbst sich dieses als ein starkes wurmwidriges Agens präsentierte, so dürfte der Schlufs Prf. Ko berts: „Nach allem Obigen mufs ich mich dahin aussprechen, dafs das ätherische Oel an der wurmtreibendon Wirkung des Filixextraktes einen wesentlichen Anteil hat" als voll berechtigt erscheinen. - Ob auch dem fotten Oele - äbnlich dem Crotonöle - Anteil an der spezifischen Wirkung zuzuschreiben ist, mul's vorläufig dahingestellt bleiben, jedenfalls wäre es aber wünschenswert, dafs durch weitere eingehende Untersuchungen die Widersprüche in den Angaben bezüglich der Wirkung der Filixsäure eine Aufklärung erführen.

Es war zunächst von Interesse zu ermitteln, aus welchen chemischen Körpern sich das atherische Filixöl zusammensetzt, um eventuell den wirkenden Bestandteil selbst zu isolieren. Bei der fraktionierten Destillation beginnt das Oel bei $140^{\circ}$ zu sieden, der Siedepunkt steigt bis $160^{\circ}$ ziemlich schnell, dann aber stetig weiter, sodafs grölsere Mengen einer bestimmten Fraktion nicht gesammelt werden konnten; es wurden erhalten von $140-180^{\circ}$ ca. 32 Proz., von $180-200^{\circ}$ ca. 20 Proz., von $200-250^{\circ}$ ca. 10 Proz., von 250$300^{\circ}$ ca. 20 Proz., im Kolben hinterbleibt dann eine schwarze, harzige Masse. Die Anteile bis zu $180^{\circ}$ haben einen ausgesprochenen Geruch nach Säuren der F'ettsäurenreihe $\mathrm{Cn}_{2 n} \mathrm{O}_{2}$, diejenigen von 180-2500 ähneln im Geruch dem Oele von Pastinaca sativa, die höheren Anteile sind augenscheinlich bereits mit brenzlichen Produkten gemischt. Das Oel reagiert stark sauer und wurde, um es zunächst von den sauren Anteilen zu befreien, mit sebr verdünnter Natriumcarbonatlösung in der Kälte behandelt, unter Kohlensäureentwickelung erhält man eine Lösung der Natronsalze der vorhan. denen Säuren.

Diese Lösung wurde von dem ungelösten Oele getrenut und zur Salzmasse eingedampft: beim Zerlegen dieser Salzmasse mit verdïnnter Schwefelsüure wurde das Gemisch der Fettsiiuren wieder abgeschieden, die eventuell noch gelösten Anteile wurden der Flüssig. 
keit durch Ausschütteln mit Aether entzogen. Es wurden auf diese Weise aus $100 \mathrm{~g}$ Filixöl $40 \mathrm{~g}$ Säuregemisch isoliert, welches bei etwa $140^{\circ}$ anfängt zu sieden; die Hauptmenge geht bei $155-165^{0}$ über, ein kleiner Teil bei $165-200^{\circ}$. Durch weitere fraktionierte Destillation liels sich aus dem Anteile $155-165^{\circ}$ eine nicht unbeträchtliche Menge eines bei $160-161^{0}$ siedenden Stoffes isolieren, welcher dem Geruche und den sonstigen Eigenschaften nach sich als Buttersäure zu erkennen gab; es liegt bier augenscheinlich ein Gemisch von Fettsäuren von Propionsäure an bis zur Capronsäure vor, in welchem die Buttersäure hinsichtlich der Menge ganz bedeutend überwiegt.

Von der Fraktion 155-1600 wurde eine $\mathrm{C}$ u. H Bestimmung vorgenommmen

$$
\begin{array}{r}
0,2638 \mathrm{~g} \text { gaben } 0,5055 \mathrm{~g} \mathrm{CO}_{2}=52,26 \text { Proz. } \mathrm{C} \\
\text { und } 0,2160 \mathrm{~g} \mathrm{H}_{2} \mathrm{O}=9,09 \\
0,2749 \mathrm{~g} \text { gaben } 0,5270 \mathrm{~g} \mathrm{CO}_{2}=52,28 \Rightarrow \mathrm{H} \\
\text { und } 0,2284 \mathrm{~g} \mathrm{H}_{2} \mathrm{O}=9,23 \Rightarrow \mathrm{H}
\end{array}
$$

Diese Zahlen liegen in der Mitte zwischen den Werten der Propionsäure und Buttersäure und deuten also auf ein Gemisch dieser beiden Säuren, es war indessen nicht möglich, durch fraktionierte Destillation ein Produkt zu gewinnen, welches mit den von Propionsäure geforderten Analysenzahlen und Siedepunkt in Uebereinstimnung zu bringen war, da die Menge dieser Fraktion zu weiterem Trennen zu gering war.

Die Fraktion 160-161 lieferte indessen Zahlen, welche mit den für Buttersïure geforderten genau übereinstimmten.

$$
\begin{array}{r}
0,321 \text { g Subst. gab } 0,6406 \mathrm{~g} \mathrm{CO}=54,42 \text { Proz. C. } \\
\text { und } 0,2664 \mathrm{~g} \mathrm{H}_{2} \mathrm{O}=9,23 \text { Proz. } \mathrm{H} . \\
0,225 \mathrm{~g} \text { Subst. gab } 0,446 \mathrm{~g} \mathrm{CO}_{2}=54,29 \text { Proz. } \mathrm{C} \\
\text { und } 0,189 \mathrm{~g} \mathrm{H}_{2} \mathrm{O}=9,37 \text { Proz. } \mathrm{H}
\end{array}
$$

$\mathrm{C}_{4} \mathrm{H}_{8} \mathrm{O}_{2}$ verlangt: 54,54 Proz. $\mathrm{C}$ und 9,11 Proz. $\mathrm{H}$.

Das aus dieser Fraktion hergestellte Silbersalz lieferte folgende Zahlen:

$$
\begin{aligned}
& 0,4996 \mathrm{~g} \mathrm{Salz} \text { gaben } 0,2761 \mathrm{~g} \mathrm{Ag}=55,26 \text { Proz. } \\
& \mathrm{C}_{4} \mathrm{H}_{7} \mathrm{Ag} \mathrm{\textrm {O } _ { 2 }} \text { verlangt: } 55,36 \text { Proz. } \mathrm{Ag} \text {. }
\end{aligned}
$$

Die Eigenschaften des Kalksalzes wiesen darauf bin, dafs das Salz der normalen Buttersäure vorlag.

Die Quantität der über $165^{\circ}$ siedenden Anteile war zu gering, um durch Destillation eine Isolierung der höheren Säuren in reinem 
Zustande za ermöglichen, doch deutet sowohl der Siedepunkt des Gemisches, als auch der Umstand, dafs selbst in der Kältemischuug ein Festwerden des Gemisches nicht beobachtet werden konnte, darauf hin, dafs höhere Süuren als etwa Capronsäure nicht vorliegen können.

Der von der verdünnten Natriumcarbonatlösung nicht veränderte Anteil wurde mit Natriumbisulfitlösung geschüttelt, es konnten indessen dem Öle keine aldehydartigen Verbindungen entzogen werden; da nun die Vermutung vorlag, dals in dem Öle Ester höherer Fettsäuren enthalten sein möchten und diese ja bei der Destillation nicht ohne Zersetzung tibergehen, das öl in der That auch beim Fortschreiten der Temperaturerhöhung im Lanfe der fraktionierten Destillation anscheinend der Zersetzung unterlag, so wurde von dieser abgesehen und zunächst durch Verseifung eine Isolierung der vorhandenen Sturen und Alkohole versucht.

$25 \mathrm{~g}$. des säurefreien Öles wurden mit $20 \mathrm{~g}$ Ätzkali und $100 \mathrm{~g}$ 50 prozentigem Alkohol einige Stunden auf dem Wasserbade am Rückflufskühler erwärnt, erst vorsịchtig der Alkohol, dann unter Erneuerung des uberdestillierenden Wassers der mit den Wasserdämpfen flüchtige Anteil abdestilliert. Auf dem wässrigen Destillat schwimmen Öltropfen, welche in ausgeprägtem Mafse den Geruch der höheren Alkohole - vornehmlich des Octylalkohols - besitzen, es wurde destilliert solange das Destillat noch deutlich roch und das gesamte destillierte Flussigkeitsquantum mit Äther ausgeschuttelt, welcher beim Abdestillieren das Gemisch der Alkohole hinterliefs.

Dieser Rückstand wurde der fraktionierten Destillation unterworfen, er begann bei $140^{\circ} \mathrm{zu}$ sieden und wurde zunăchst in Intervallen von $20^{\circ}$ aufgefangen, die letzten Anteile gingen bei etwa $250^{\circ}$ uber, oberhalb dieser Temperatur fand augenscheinlich Zersetzung statt. Durch weiteres Fraktionieren wurden reinere Anteile gewonnen von $155-160^{\circ}$ und von $190-195^{\circ}$.

Die Fraktion $155-160^{\circ}$ gab bei der Analyse Zahlen, welche mit den für Hexylalkohol geforderten nahe Übereinstimmung zeigen:

0,1813 g gaben 0,4711 g CO$_{2}=70,860$ Proz. C und $0,2107 \mathrm{~g} \mathrm{H} \mathrm{H}_{2} \mathrm{O}=12,012$ Proz. $\mathrm{H}$

$\mathrm{C}_{6} \mathrm{H}_{14} \mathrm{O}$ berechnet: $\mathrm{C}=70,55$ Proz. und $\mathrm{H}=13,75$ Proz.

Die Fraktion 190-195, welche sich dem Geruche nach entArub. d. Pharm. CcxxrI. Bda s. Heft. 
scheiden als Octylalkohol präsentierte, gab bei der Analyse weniger gut stimmende Zahlen; es rülurt dies daher, dals über $200^{\circ} \mathrm{K}$ orper übergehen, welche einen höheren Koblenstoffgehalt besitzen und da sie auch in den Stadien $190-195^{\circ}$ zweifellos schon mit enthalten sind, den Wasserstoffgehalt herabdrücken. Es ist indessen besonders bei der geringen Menge des zur Verfügung stehenden Materials nicht möglich durch fraktionierte Destillation eine weitere Reinigung herbeizuführen.

Die Fraktion gab folgende Analysenzahlen:

$$
\begin{array}{r}
0,2232 \mathrm{~g} \text { gaben } 0,6014 \mathrm{~g} \mathrm{CO}=73,48 \text { Proz. } \mathrm{C} \\
\text { und } 0,2587 \mathrm{~g} \mathrm{H}_{2} \mathrm{O}=12,90 \text { Proz. } \mathrm{H}
\end{array}
$$

Für $\mathrm{C}_{8} \mathrm{H}_{18} \mathrm{O}$ ist berechnet 73,85 Proz. $\mathrm{C}$ und 13,85 Proz. $\mathrm{H}$.

Die höheren Fraktionen geben bei der Analyse Zahlen, welche in die Reihe der Alkohole $\mathrm{C}_{n} \mathrm{H}_{2 \mathrm{n}}+{ }_{2} \mathrm{O}$ überhaupt nicht passen, sondern eine bedeutende Abnahme des Wasserstoffgehaltes zeigen; dieselben geben der Reihe nach Zahlen, wie $\mathrm{C}=79,65 \% \mathrm{H}=10,87 \%$ und $\mathrm{C}=81,41 \% \mathrm{H}=10,95 \%$, was etwa Verbindungen der Formel $\mathrm{C}_{11} \mathrm{H}_{18} \mathrm{O}$ und $\mathrm{C}_{14} \mathrm{H}_{23} \mathrm{O}$ entsprechen würde. Es scheint, dals hier Vertreter der aromatischen Reihe, vielleicht Cineol oder Verwandte der Terpenreihe vorliegen und bin ich gegenwärtig beschätitigt nach Darstellung einer grölseren Menge des Öles die Natur dieser Verbindungen aufzuklären.

Der alkalische Rtickstand in dem Kolben, aus welchem die Alkohole etc. abgetrieben waren, wurde nach Verdünnen mit Wasser. ehenfalls mit Äther ausgeschüttelt, es wurde auf diese Weise eine מufserst geringe Menge eines Öles erhalten, welches augenscheinlich von dem durch Destillation mit Wasser gewonnenen Alkoholgemisch nicht verschieden war. - Dieser alkalische Rückstand wurde sodann mit verdünnter Schwefelsäure übersättigt und wieder mit $\ddot{A}$ ther ausgeschüttelt, das nach dem Abdestillieren des Äthers verbleibende Fettsäuregemisch wurde der Destillation mit Wasserdampf unterworfen, um die flüchtigen von den nicht flüchtigen Fettsäuren zu trennen. Das Destillat enthielt eine grölsere Menge Stuure gelöst, während eine geringere Menge als Öltropfen aufschwamm; letztere wurde für sich vorsichtig gesammelt und über das Ammonsalz in das Silbersalz verwandelt.

Bei der Analyse dieses Salzes wurden Zahlen erhalten, welche 
darauf hindeuten, dafs hier Pelargonsäure, vermischt mit Spuren von Caprylsăure vorliegt.
$0,4185 \mathrm{~g}$ Silbersalz gaben $0,1729 \mathrm{~g} \mathrm{Ag}=41,31$ Proz.
Pelargonsaures Silber enthält 4(',78 Proz. Ag.
Caprylsaures
43.06 Proz. Ag.

Beim Ausschütteln des Destillationsrückstandes in der Retorte erhält man eine kleine Menge einer Fettsäure, welche indessen zu weiterer Untersuchung zu gering war; da die Pelargonsaure gerade die Grenze bildet zwischen den noch mit Wasserdämpfen flüchtigen und den nicht flüchtigen Säuren, so dürfte wohl hier nur ein Anteil von dieser Säure zurückgeblieben sein.

Aus dem wässerigen Destillat wurden die Säuren mit Äther ausgeschüttelt und nach Abtreiben dieses der Destillation unterworfen, es konnte auch hier wieder mit Sicherheit Buttersäure nachgewiesen werden, während die höheren Săuren - da in geringerer Menge vorbanden, nicht in analysierbarem Zustande gewonnen werden konnten. Dals indessen höhere Săuren bis zur Caprylsăure jedenfalls vorhanden sind, geht daraus hervor, dals die höher siedenden Anteile bei starker Winterkälte zum Erstarren zu bringen sind.

Aus den vorlkufig gewonnenen Anhaltspankten geht hervor, dals das Filixól zum Teil aus freien Fettsänren besteht, unter denen die Buttersäure vorherrscht, dafs ferner eine Reihe von Estern der Fettsäurereihe von der Buttersăure an aufwärts bis etwa zur Pelargonsture des Hexyl- and Octylalkohols vorhanden sind und dalis susserdem noch augenscheinlich Angehörige der aromatischen Reibe in geringeren Mengen nachgewiesen werden können. - Dafs die Fettsäuren in der Wurzel bereits frei enthalten sind und nicht lediglich als ein durch die Einwirkung des Wasserdampfes gebildetes Spaltungs- resp. Verseifungsprodukt anzusehen sind, zeigt uns die stark saure Reaktion der Wurzel selbst. Diese Säuren sind ja für die anthelmintische Wirkang des Extraktes wohl nicht verantwortlich zu machen, ob die Wirkung nun auf Rechnung der höheren Ester, oder - was vielleicht wahrscheinlicher - auf Rechnung der noch anwesenden aromatischen Substanzen zu setzen ist, sollen weitere Versuche, mit denen ich zur Zeit beschăftigt bin, ergeben.

Ich bemerke hier noch zum Schlusse, dafs auch ein atherisches Extrakt aus der Panna-Wurzel (Wurzel von Aspidium athamanticum), 
welches ich herstellte, sich nach den Untersuchungen des Herm Prof. Kobert als wurmfeindlich orwies und dafs auch hier ein von ätherischem Öle befreites Extrakt eine bedeutend schwächere Wirkung zeigte, als das nicht von Öl befreite Präparat.

Erfurt, Dezember 1892.

\title{
Über die Verwendung von Baryumhydroxyd in der Butteranalyse
}

\author{
von E. Laves in Strafsburg i. E.
}

(Eingegangen den 10. IV. 1893.)

Die Reichert-Meissl 'sche Zahl schwankt bekanntlich für reines Butterfett zwischen 22 und 34. Infolge dieser grolsen Schwankung der Werte für reines Butterfett ist diese Methode in vielen Fullen durchaus unzureichend, um fremde Fetto im Butterfett nachzuweisen. Um diese Lücke auszufüllen, mufste man eine Methode ausfindig zu machen suchen, durch welche Fettsäuren der Butter bestimmt werden, welche in den verschiedenen Butterarten in einem möglichst konstanten, von anderen Fetten stark abweichenden Verhältnis vorkommen. Aufserdem mufste diese Methode eine exaltere Trennung der betr. Fettsäuren ermöglichen, als dies durch Destillation möglich ist.

Es lag am nächsten, die Trennung der Fettsäuren in Form ihrer Salze auszufuhren und zwar ihrer Baryum-oder ihrer Bleis alze.

v. Raumer versuchte, die Menge der Ölsäure in Butterfett durch Extraktion der fettsauren Bleisalze mit Äther zu bestimmen, fand aber sehr schwankende Werte.

Die Löslichkeit der fettsauren Baryumsalze lassen dieselben sehr geeignet zur Trennung der Fettsäuren in der Butter erscheinen, da die Baryumsalze der niedern Fettsäuren in Wasser und verdünntem Alkohol leicht löslich, die der höhein Fettsäuren (von der Capronsäure aufwärts) aber fast unlöslich sind. Bei Anwendung von Baryumhydroxyd kann dasselbe zugleich als Verseifungsmittel des Fettes dienen. In dieser Richtung sind verschiedene Versuche gemacht worden. 